\title{
Measuring health-related quality of life in patients with advanced cancer: a systematic review of self-administered measurement instruments
}

\author{
Janneke van Roij ${ }^{1}$ Heidi Fransen ${ }^{1} \cdot$ Lonneke van de Poll-Franse $^{1,2,3} \cdot$ Myrte Zijlstraa $^{1,4} \cdot$ Natasja Raijmakers ${ }^{1}$
}

Accepted: 5 February 2018 / Published online: 10 February 2018

(c) The Author(s) 2018. This article is an open access publication

\begin{abstract}
Purpose Patient-reported outcome measures (PROMs) are becoming increasingly important in clinical practice. The implementation of PROMS in routine practice is challenging because information regarding psychometric quality of measurement instruments is fragmented and standardization is lacking. The aim of this study is to evaluate the quality of self-administered HRQoL measurement instruments for use in patients with advanced cancer in clinical practice.

Methods A systematic literature search was performed in PubMed, Embase, PsycINFO, and CINAHL to identify studies concerning self-administered HRQoL measurement instruments in patients with advanced cancer between January 1990 and September 2016. Quality of the measurement instruments was assessed by predefined criteria derived from the COSMIN checklist.

Results Sixty-nine articles relating to 39 measurement instruments met the inclusion criteria. Information regarding important measurement properties was often incomplete. None of the instruments performed sufficient on all measurement properties. Considering available information, the EORTC QLQ-C15-PAL appeared to have adequate psychometric properties, together with the EORTC QLQ-BM22.

Conclusions Many of the existing HRQoL measurement instruments have not yet been evaluated in an adequate manner. Validation of self-administered HRQoL measurement instruments is an ongoing development and should be prioritized. This review contributes to improved clarity regarding the availability and quality of HRQoL measurement instruments for patients with advanced cancer and supports health care professionals in an adequate selection of suitable PROMs in clinical practice.
\end{abstract}

Keywords Quality of Life $\cdot$ Medical oncology $\cdot$ Palliative care $\cdot$ Validation studies $\cdot$ Patient-reported outcomes

\section{Introduction}

Electronic supplementary material The online version of this article (https://doi.org/10.1007/s11136-018-1809-4) contains supplementary material, which is available to authorized users.

Janneke van Roij

j.vanroij@iknl.nl

1 The Netherlands Comprehensive Cancer Organisation, PO Box 19079, 3501 DB Utrecht, The Netherlands

2 CoRPS - Center of Research on Psychology in Somatic diseases, Department of Medical and Clinical Psychology, Tilburg University, Tilburg, The Netherlands

3 Division of Psychosocial Research and Epidemiology, The Netherlands Cancer Institute, Amsterdam, The Netherlands

4 Department of Medical Oncology, Radboud MC, Nijmegen, The Netherlands
Integration of palliative care in oncology is recommended by European Society for Medical Oncology (ESMO) and American Society of Clinical Oncology (ASCO) as oncological palliative care will enhance quality of life (QoL), and may also positively influence the course of illness [1]. In their landmark paper, Temel et al. showed that early palliative care in fact leads to significant improvements in both healthrelated quality of life (HRQoL) and mood [2]. For high-quality oncological palliative care in advanced cancer patients it is essential to monitor HRQoL in clinical practice in a suitable manner [3]. HRQoL generally consists of four domains: physical well-being, psychological well-being, social wellbeing, and spiritual well-being. Especially the spiritual wellbeing is important in patients with advanced cancer due to the confrontation with death [4-12]. Monitoring symptoms 
and HRQoL is extremely important in advanced cancer care, because it increases awareness among health care professionals to better anticipate on patients' changing needs [13, 14] and improves clinical outcomes (i.e. fewer emergency room visits, fewer hospitalizations, a longer duration of palliative chemotherapy, and superior quality-adjusted survival), as recently demonstrated by Basch et al. [14].

The best method to monitor HRQoL in patients is to ask patients themselves, as asking health professionals or relatives is considered a less accurate method for estimating the HRQoL of a patient [15]. Inclusion of patient-reported outcome measures (PROMs) in routine clinical practice is, beside clinical benefits, also associated with improvements in discussion of patient outcomes during consultations and patient satisfaction [16-18]. However, the implementation of PROMS in routine practice is challenging because information regarding psychometric quality of measurement instruments is fragmented and standardization is lacking [19].

Earlier reviews have identified a variety of HRQoL measurement instruments that were appropriate for use in oncological palliative care [20-27]. However, none of these reviews could serve as a guide for an adequate and comprehensive choice of a measurement instrument for routine clinical practice because none used explicit criteria assessing measurement properties. For this reason, in 2010 Albers et al. [28] made an inventory of available HRQoL measurement instruments that were suitable for the use in palliative care and assessed the quality of these instruments. This review identified 29 different measurement instruments and showed a wide variety in measurement aim, content, target population, method (e.g. interview, questionnaire), completion time/length, and clinimetric quality [28]. In the last six years, a growing body of research has been published on the quality of existing HRQoL measurement instruments and also the development of new instruments is ongoing. It remains unclear what PROMs are most suitable for advanced cancer patients, receiving oncological palliative care nowadays.

Because the measurement of HRQoL in advanced cancer patients is a rapidly evolving field and the importance of PROMs in clinical practice is growing, an updated review on HRQoL measurement instruments seems appropriate. The aim of this study is to evaluate the quality of selfadministered instruments measuring HRQoL of patients with advanced cancer for use in oncological palliative care nowadays. The methodological quality of the measurement instruments is described in terms of measurement properties and measurement quality. This review aims to contribute to more clarity regarding the availability and quality of selfadministered HRQoL measurement instruments for patients with advanced cancer and to support health care professionals in an adequate selection of suitable PROMs in advanced cancer patients in clinical practice.

\section{Methods}

\section{Search strategy}

An electronic search of the database PubMed, Embase, PsycInfo, and CINAHL was performed to identify papers about instruments to measure HRQoL in advanced cancer patients that were published in English or Dutch between January 1990 and September 2016. Non-validation studies (article type) were excluded. A search strategy was developed for finding relevant publications in electronic literature databases, based on the search strategy of Albers et al. [28]. The computerized search was conducted using a search strategy to find studies on HRQoL measurement instruments in oncological palliative care: 'palliative', 'instruments', and 'QoL'. A detailed description of the MeSH-terms and keywords used in the search can be found in Supplement 1. The search string was initially developed in PubMed and later adapted for the other databases. Additionally, all Validation Studies (article type) of the 29 identified HRQoL measurement instruments of the review of Albers et al. [28] were added. In addition, the reference lists of selected articles were screened to retrieve relevant publications which had not been found in the computerized search.

\section{Study selection process}

Two reviewers (NR and HF) used a stepwise procedure to identify relevant studies. Firstly, all papers' titles and abstracts were assessed for relevance by one of the reviewers (NR) to see if the study describes the development or validation of a measurement instrument and whether the study involves (at least two domains of) HRQoL as outcome measurement. Irrelevant titles were excluded. Secondly, abstracts were screened by two reviewers (NR and HF) on the following inclusion criteria: (i) the study concerned the development or validation of a self-administered measurement instrument; (ii) non-primary tumour-specific HRQoL (and at least two of its domains) was a primary or secondary objective of the study; (iii) the target population of the study included adult patients (i.e. $\geq 18$ years old) with advanced or metastatic cancer; (iv) the measurement instrument used in the study was provided in Dutch or English language; (v) only full-text English or Dutch reports were included. Consensus regarding exclusion based on these exclusion criteria was reached after a consensus meeting. Of all the studies that did not pass the selection process, the reasons for exclusion were listed. Full-text papers were also assessed on the above-mentioned criteria and conference abstracts were excluded. 


\section{Data extraction procedure}

Two reviewers (NR and JvR) independently reviewed five randomly selected papers using a standard data extraction sheet and compared results to evaluate uniformity. Then, all papers were divided between the two researchers (NR and JvR) for data extraction. The procedure to confirm uniformity was repeated three times during the data extraction phase.

The methodological quality of included validation studies was assessed using the COnsensus-based Standards for the selection of health Measurement INstruments (COSMIN) checklist devised by Mokkink et al. [29]. Supplement 2 gives an overview and a description of the criteria used to assess quality. The assessment for the methodological quality of studies on measurement properties of health status measurements instruments covers nine topics: internal consistency, reliability, measurement error, content validity, construct validity (i.e. structural validity, hypotheses testing, and cross-cultural validity), criterion validity, and responsiveness. The methodological quality of the selected publications was assessed by two researchers (NR and JvR). The quality assessment was evaluated in the same manner as described earlier.

\section{Results}

\section{Selection of papers}

A flowchart of the selection process is presented in Fig. 1. In total, 4088 articles were identified from the different electronic databases, excluding duplicates. Initially, 3854 papers were excluded based on screening of relevance of title and abstract. The abstracts of the remaining 234 articles were assessed in depth for eligibility by two researchers (NR and HF). Finally, 126 studies were suitable for fulltext assessment. During full-text assessment, 37 studies were excluded. A number of studies $(n=11)$ were excluded because no full text was available after multiple attempts to retrieve the paper by contacting the author via Research gate or Email. Of these 11 papers, three were published more than 10 years ago, six were published in low-impact journals (impact factor $<2$ ), which were often less accessible and two were untraceable. Other papers were excluded if they were a congress abstract $(n=14)$, the measurement instrument used in the study was in a language other than Dutch or English $(n=2)$, it was a duplicate $(n=4)$, it was not a self-administered measurement instrument $(n=4)$, it was not an measurement instrument $(n=2)$, or the measurement instrument was unidimensional or disease specific $(n=29)$. After checking reference lists of the selected articles, nine additional articles were identified. In total, 69 papers were included in this systematic review.

\section{Study characteristics}

The selected studies had between 10 and 3282 participants (21,077 participants in total) of whom 22-99\% were men. Across studies, the average age of participants ranged from 51 to 79 years. Twenty percent of the studies included palliative patients suffering from various life-threatening illnesses (e.g. heart failure, end-stage lung disease, advanced renal disease, late-stage Parkinson disease, cancer), with the majority suffering from advanced cancer. Other studies focussed on cancer patients of which most studies (67\%) included a mixed cancer population (i.e. various primary cancer sites). The remaining studies (13\%) selected one specific primary cancer site: $4 \%$ patients with lung cancer, $3 \%$ women with breast cancer, $3 \%$ patients with brain tumours, $1 \%$ men with prostate cancer, and $1 \%$ patients with colorectal cancer.

\section{Health-related quality of life measurement instruments}

Table 1 gives an overview of all the measurement instruments that were included in this review including the full form of the used acronyms. Across studies 39 measurement instruments were identified. Instruments were originally developed between 1972 (General Health Questionnaire -12) and 2013 [European Organisation of Research and Treatment of Cancer (EORTC) Quality of Life Questionnaire (QLQ)-Social Well-being 36]. The EORTC QLQ Core 30 (EORTC QLQ-C30) was most popular because ten studies $(14 \%)$ validated this measure and seven studies $(10 \%)$ administered a module of the EORTC (i.e. QLQ-Bone Metastases module 22 (QLQ-BM22), QLQ-Brain module 20 (QLQ-BN20), QLQ-Oral Health 17 (QLQ-OH17), and QLQ-SWB36). Nine studies (13\%) validated the Edmonton Symptom Assessment Scale (ESAS) (or a modified or revised version of the ESAS), seven studies (10\%) used the McGill Quality of Life Questionnaire (MQOL) (or the revised version), and four (6\%) studies validated the Palliative care Outcome Scale (POS). For the majority of the measurement instruments (58\%) they measure (HR)QoL, eight instruments (11\%) with symptom assessment or the impact of symptoms on daily functioning. For other measurement instruments it is argued that they assess spiritual well-being or spiritual distress (14\%), psychological disorders or depressive symptoms (5\%), core concerns and palliative needs $(2 \%)$, or parenting concerns for adults with cancer $(2 \%)$.

The number of items the measurement instruments contained ranged between one [Minimal Documentation System (MIDOS) and Quality of Life in Life-Threatening Illness-Patient version (QOLLTI-P)] and 106 [Resident Assessment Instrument for Palliative Care (RAI-PC)]. The 


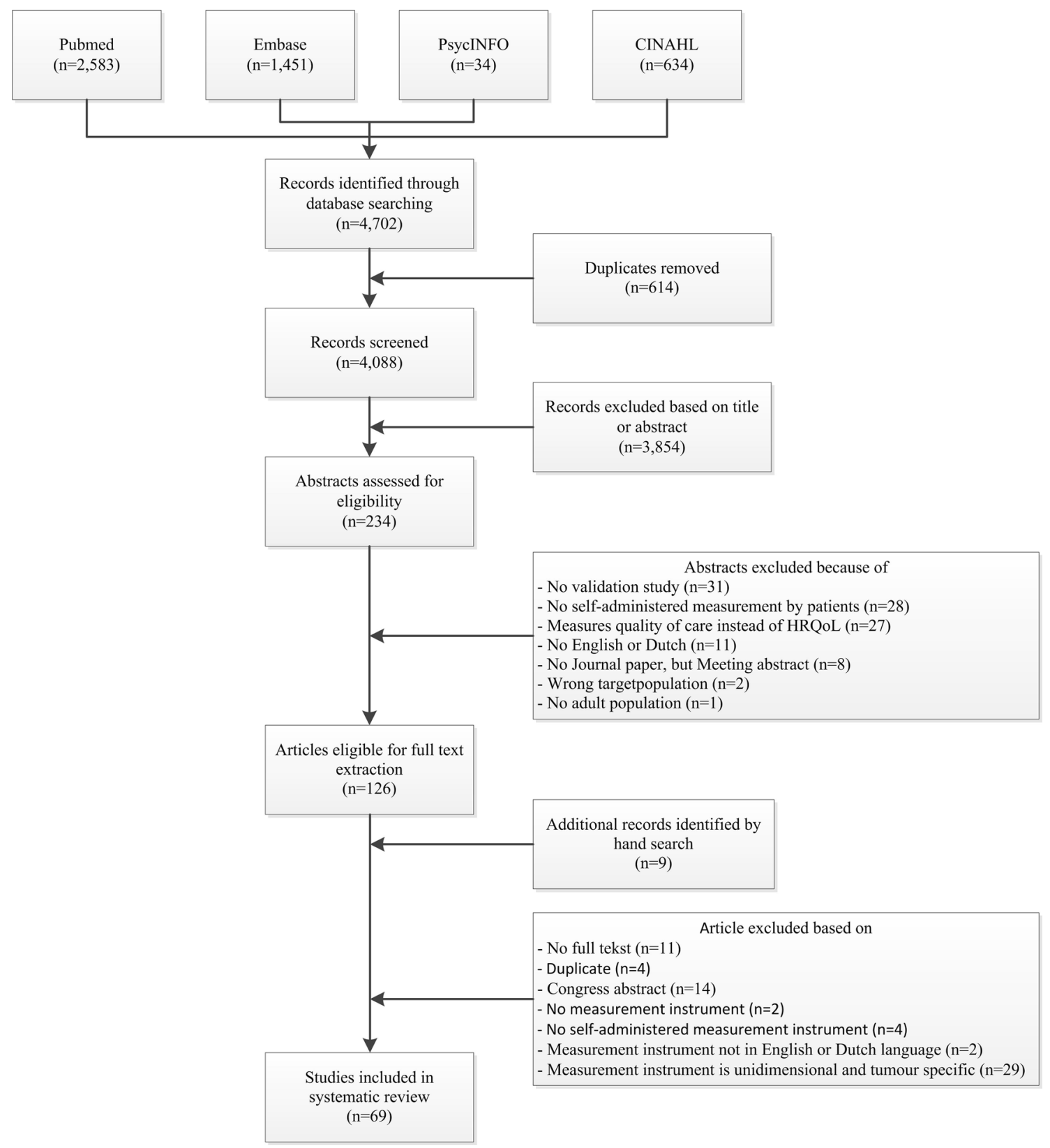

Fig. 1 Flowchart study process

scoring of the measurement instruments was most often calculated as a total score and a subscale score $(44 \%)$ or merely subscale scores $(19 \%)$ or only a total score $(14 \%)$. Other measurement instruments used single-item scores (5\%), or a combination of single (visual analogue scale) items, subscale, and a total score (12\%). One measurement instrument (2\%) used content analysis to analyse responses.

Eight measurement instruments (19\%) focused on the general population or patients in general, nine $(21 \%)$ were targeted at palliative patients, nine $(21 \%)$ at patients with cancer, and eight (19\%) at patients with advanced cancer in specific. The target population of four measurement instruments $(9 \%)$ were patients with brain tumours or brain metastases in specific. The remaining measurement instruments $(12 \%)$ focused on bone or spina metastases, chest malignancies in cancer patients, and anorexia or cachexia. Most measurement instruments (33\%) had a recall time of one week or had no recall time (14\%). Others used a recall time of three days (7\%), two weeks (2\%), one month (2\%), or one day $(2 \%)$. The completion time of seven measurement instruments (16\%) was reported. The completion time 


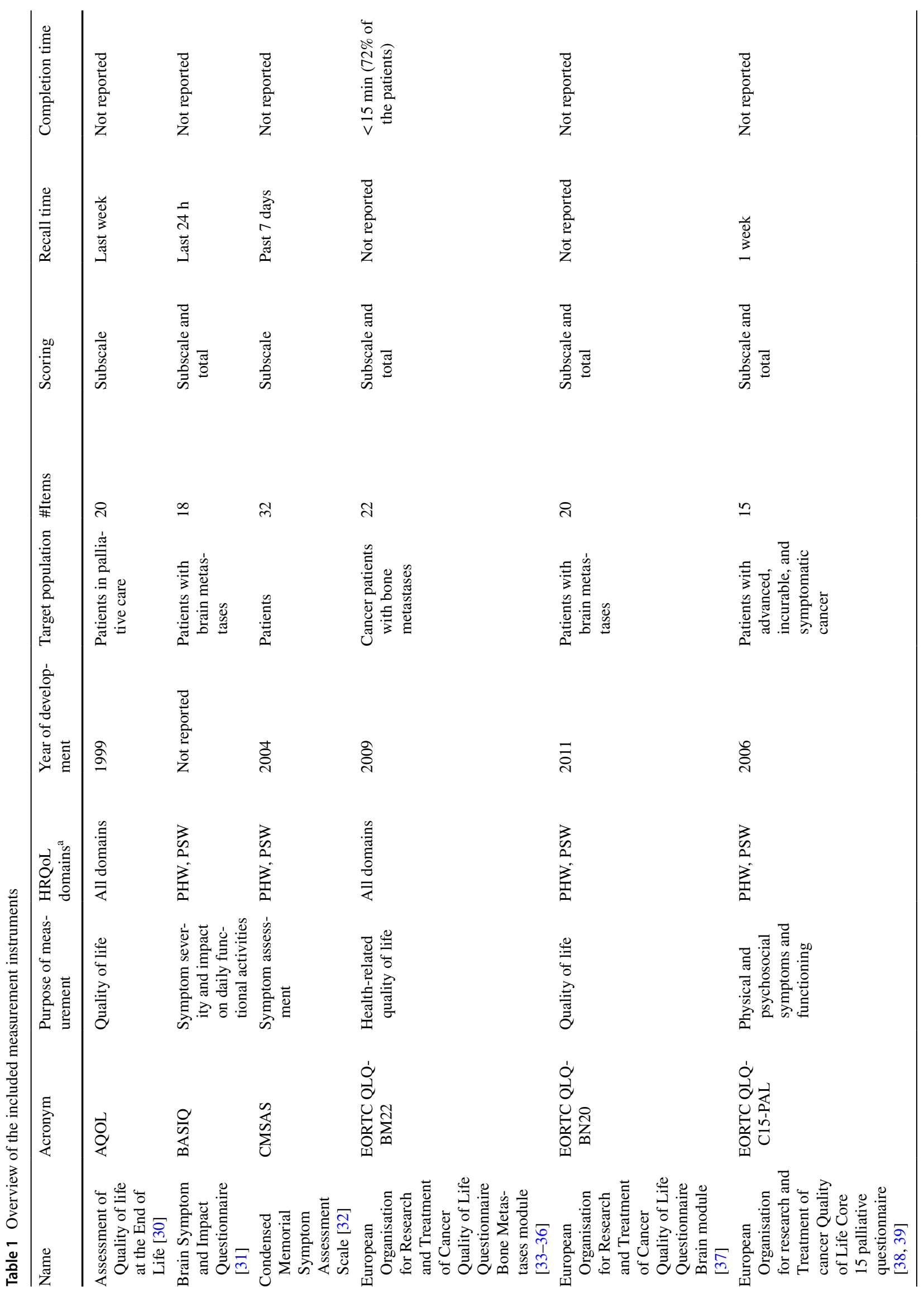




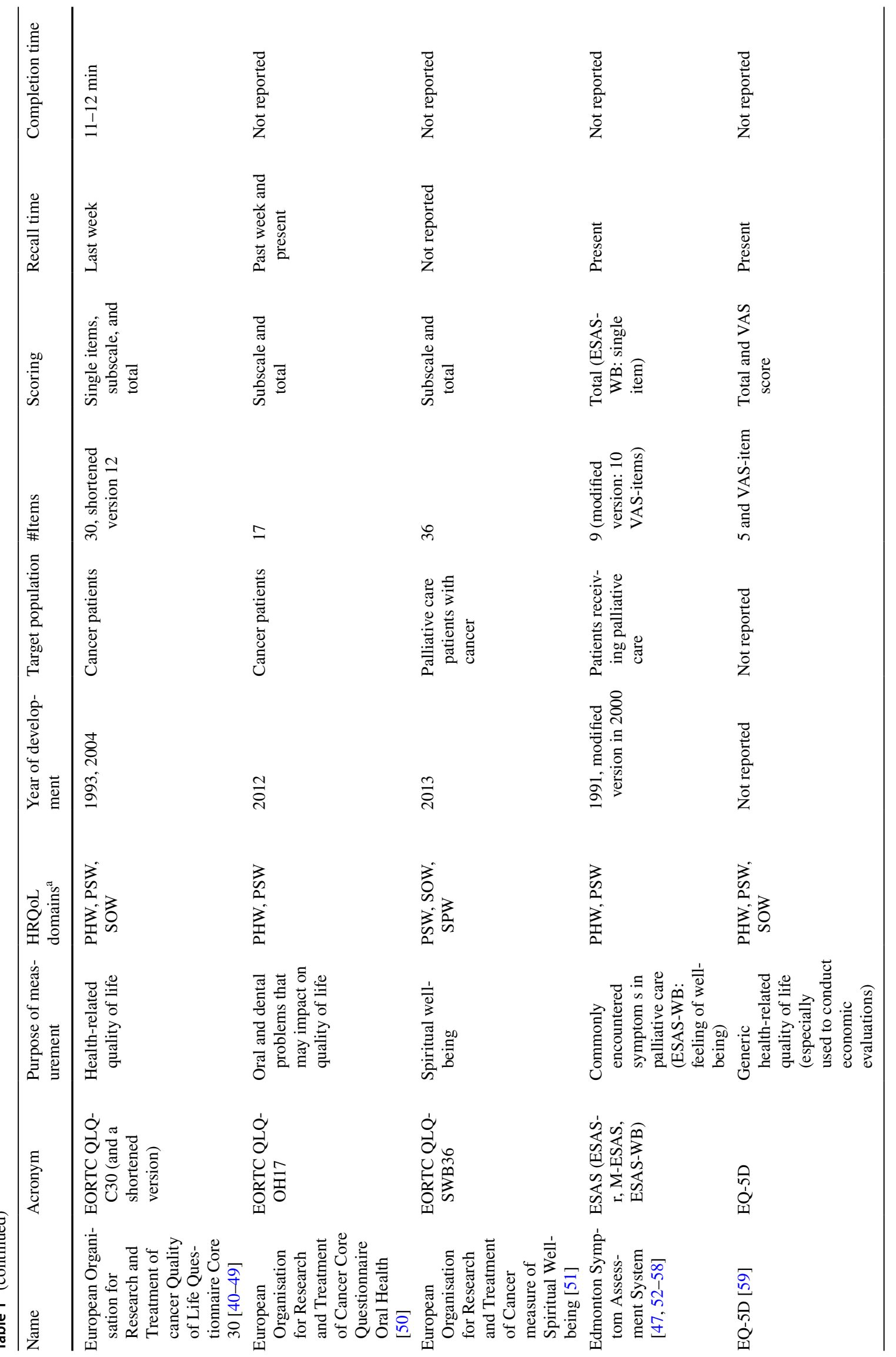




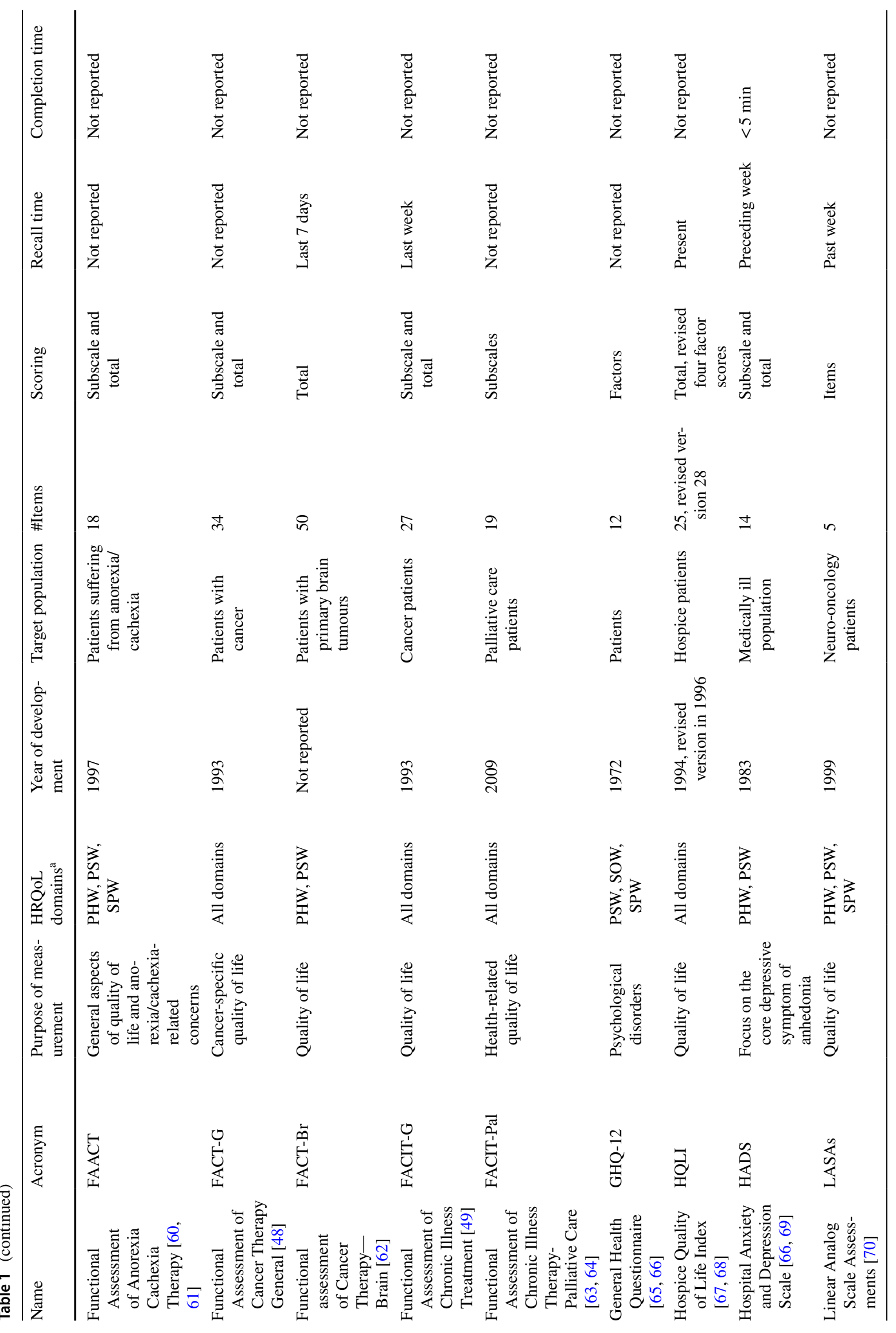




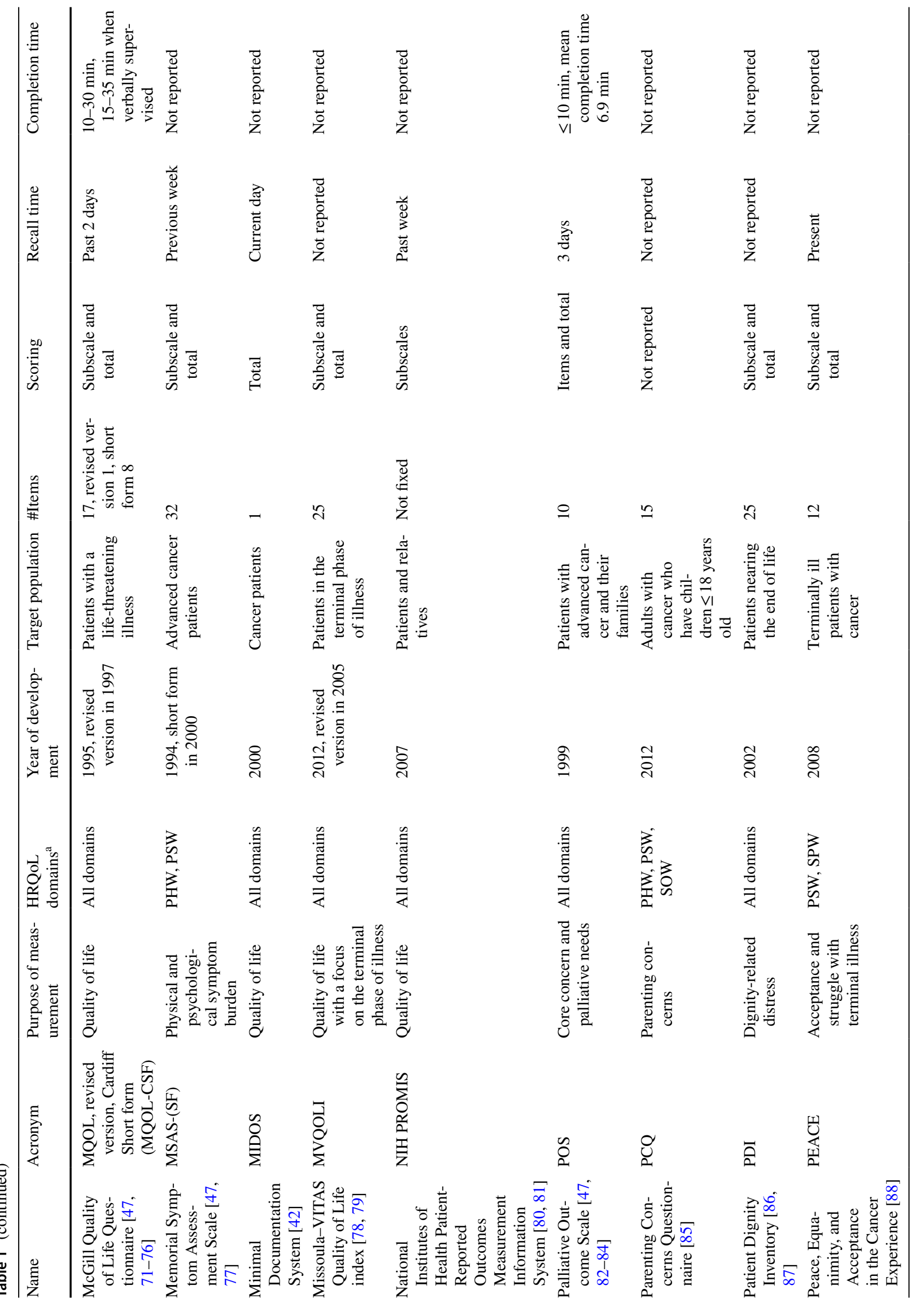




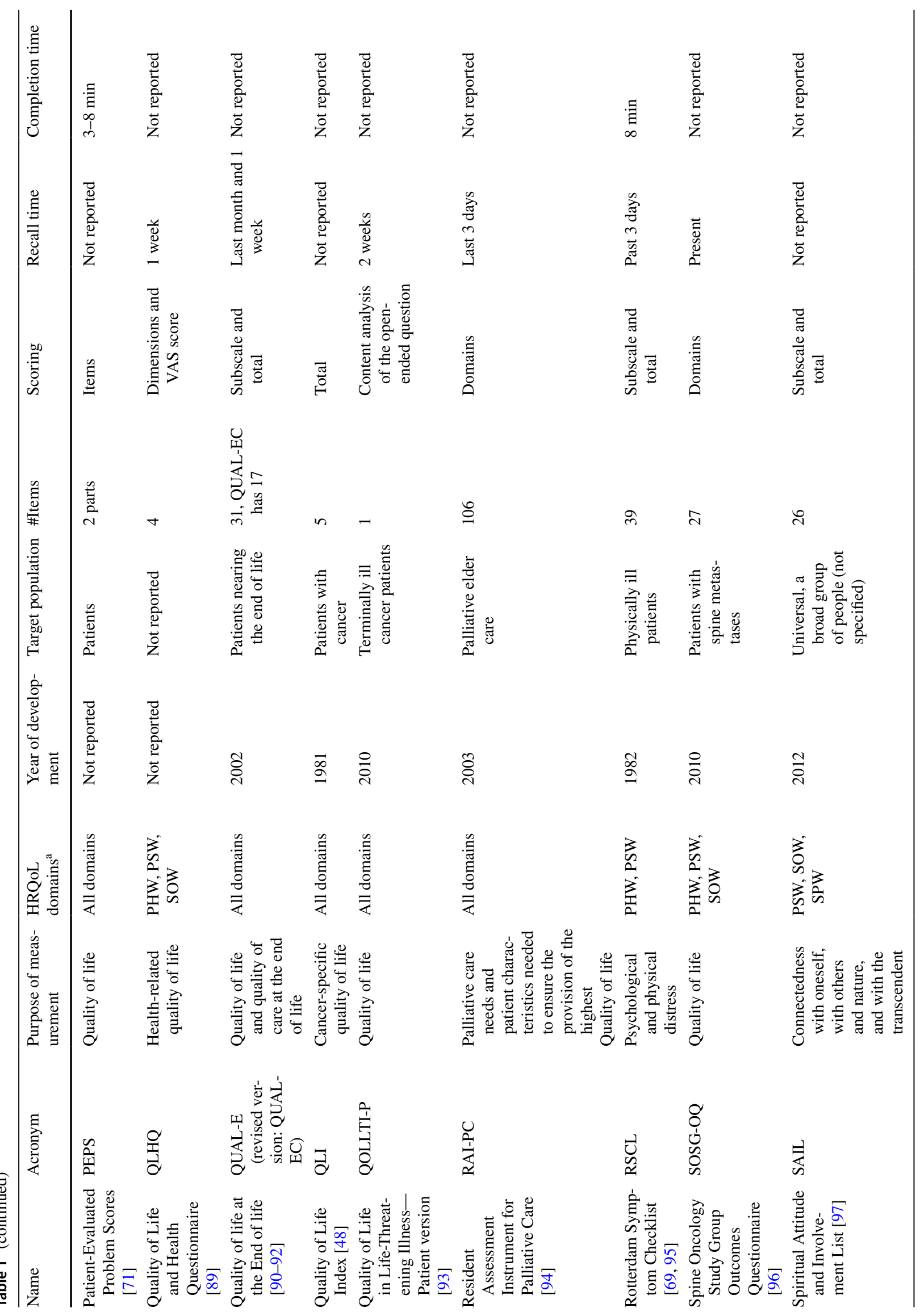


ranged between three [Patient-Evaluated Problem Scores (PEPS)] to $30 \mathrm{~min}$ (MQOL).

\section{Measurement properties}

None of the measurement instruments were adequately assessed for all measurement properties (Table 2). Information about the content validity (94\%) was most often reported and in most cases adequate (58\%). Information on the construct validity was reported by the majority of the studies (70\%). However, compared to other measurement properties, the construct validity was most often inadequately tested (30\%). Furthermore, information about the absolute measurement error, responsiveness, and interpretability was often incomplete $(6,22$, and $51 \%$ respectively) or completely missing $(88,74$, and $46 \%$ respectively).

Considering the available information on measurement properties, the EORTC QLQ Core 15 palliative questionnaire (QLQ-C15-PAL) showed best results. For instance, the EORTC QLQ-C15-PAL showed good content and construct validity, and the absolute measurement error and interpretability was also good. Other measurement properties had not been tested for the EORTC QLQ-C15-PAL. Equivalently, the EORTC QLQ-BM22 also appeared to have adequate psychometric properties because it appeared to have a good content and construct validity and the measurement instrument is reliable and responsive.

The ESAS showed good content validity, and the absolute measurement error and interpretability was good. However, information was lacking on other measurement properties. Other measurement instruments that had reasonable psychometric properties were the Assessment of Quality of life at the End of Life (AQOL), Quality of life at the End of life (QUAL-EC), and the Spiritual Attitude and Involvement List (SAIL). They had good content and construct validity, the internal consistency was good, but other information on measurement properties was lacking or missing.

The EORTC QLQ-C30 had undergone the most validation studies compared to other instruments but the studies did not adequately evaluate some important fundamental psychometric properties. The content validity, construct validity, and absolute measurement error of the EORTC QLQ-C30 was good. Evidence on other psychometrics characteristics of the EORTC QLQ-C30 was unclear.

The POS, QUAL-E, and MQOL were also tested by multiple studies. The POS had good content validity and construct validity, but the internal consistency was inadequate. Information on other measurement properties was lacking or missing. The QUAL-E showed a good content validity and construct validity. However, the internal consistency and reliability was inadequately tested and information on other measurement properties was incomplete. The revised version 


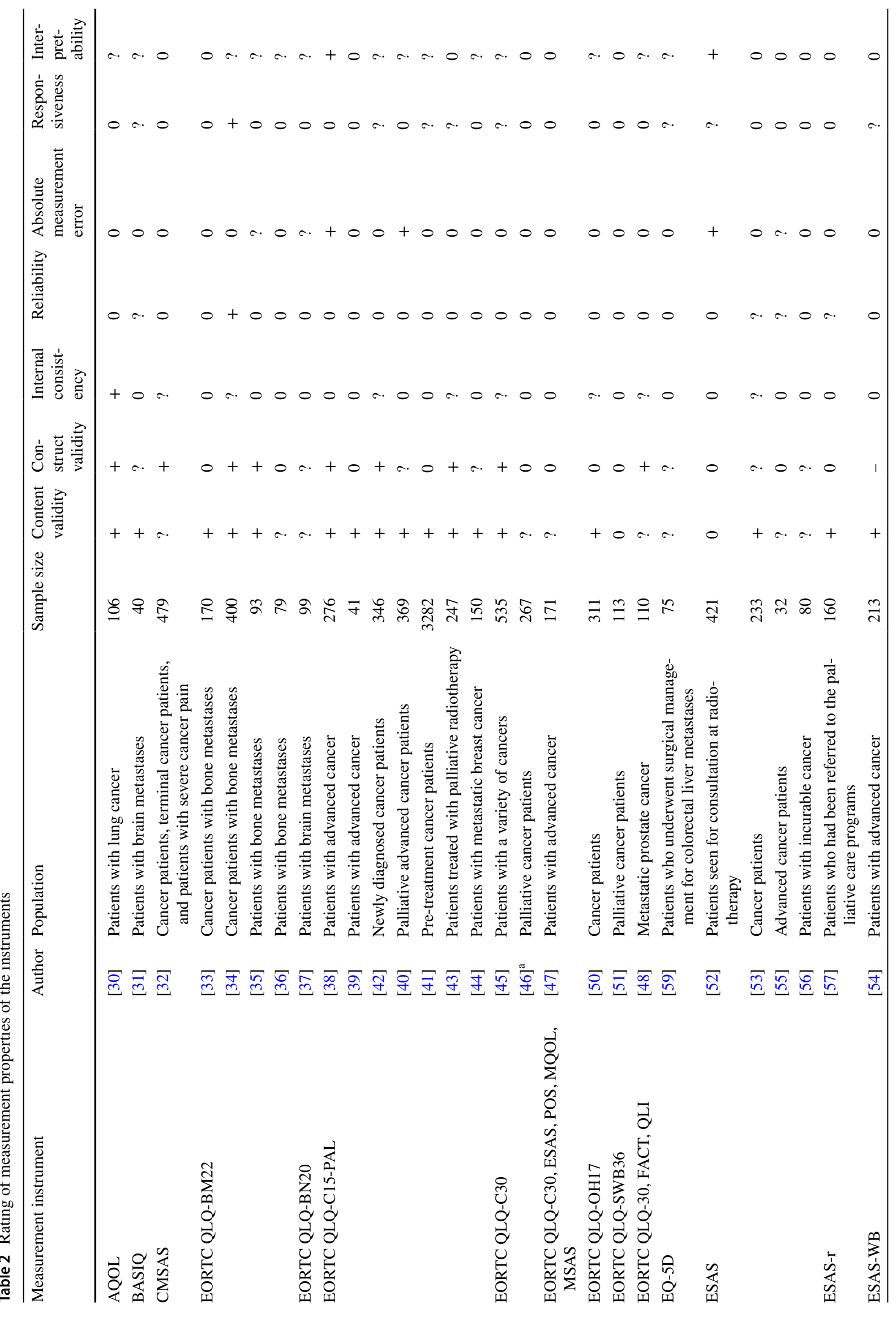




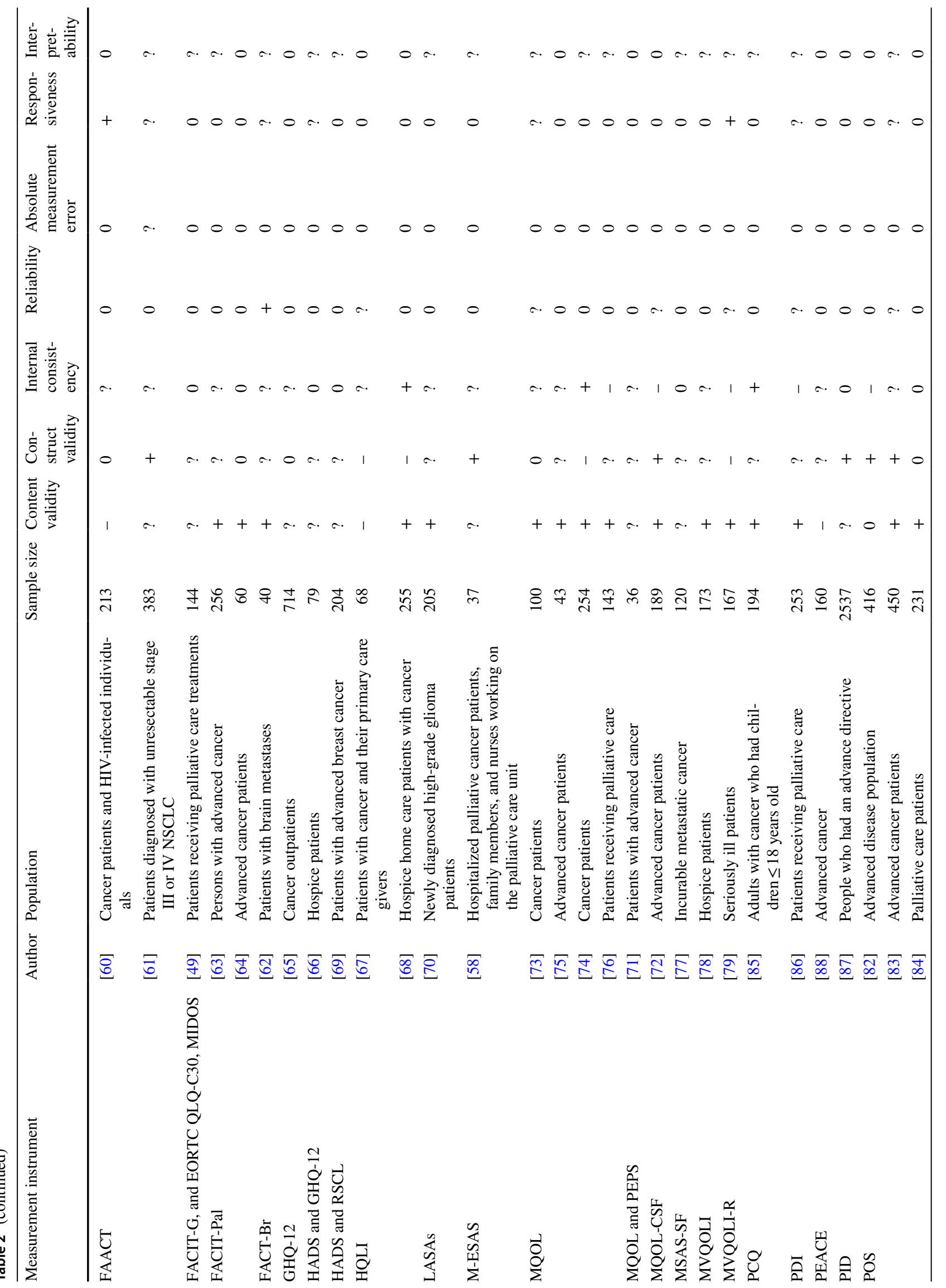




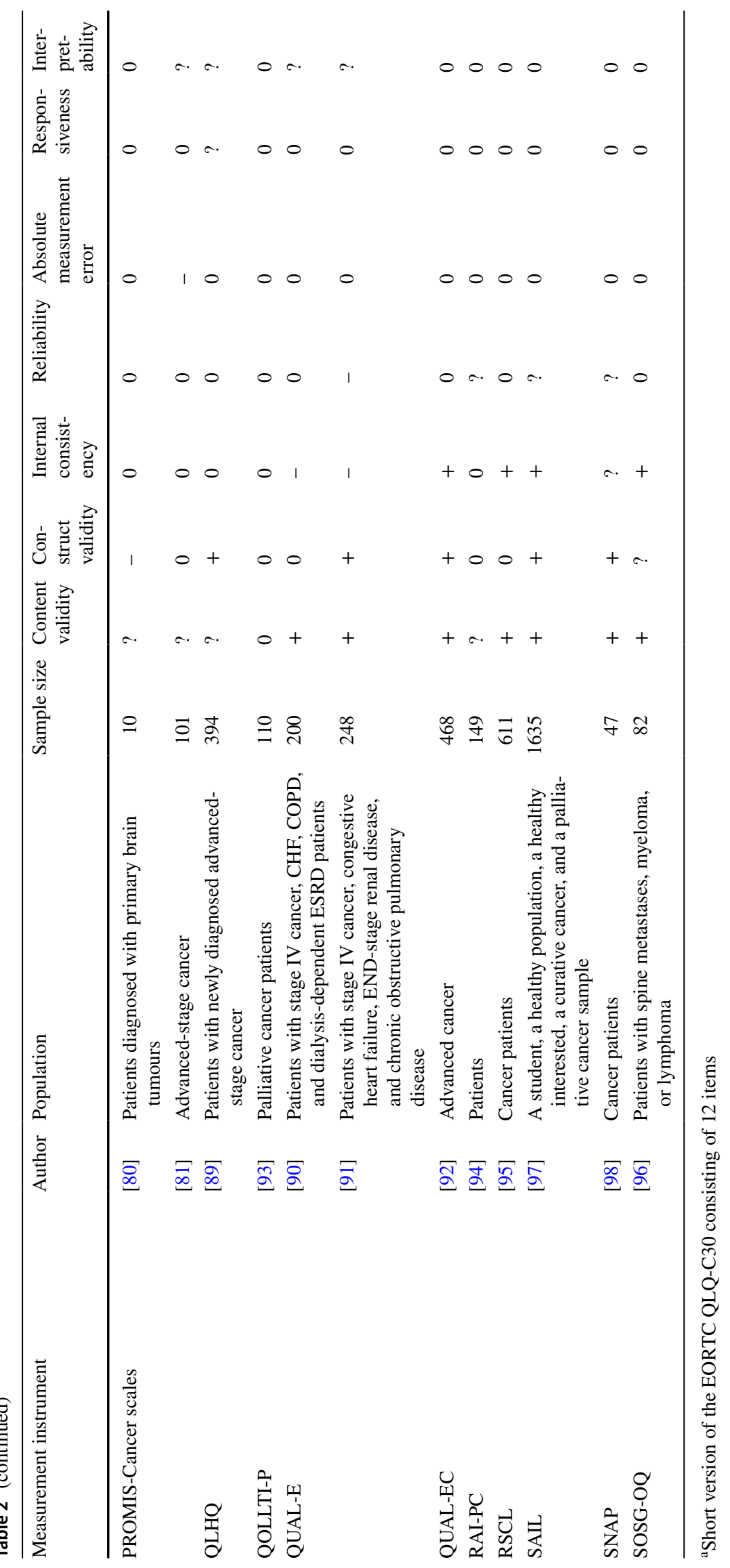


of the QUAL-E (QUAL-EC) showed improved measurement properties. The MQOL had adequate content validity, but inadequate construct validity. There was conflicting evidence regarding the internal consistency of the MQOL, and other measurement properties were inadequately tested.

There was consensus across two studies that the Hospice Quality of Life Index (HQLI) had inadequate construct validity. Results about the content validity were inconsistent, the internal consistency of the measurement instrument was good, and other psychometric information was lacking. For the EORTC QLQ-SWB36 and the QOLLTI-P, information on any of the measurement properties was absent. Other measurement instruments such as the EORTC QLQ-BN20, EQ-5D, Functional Assessment of Chronic Illness Treatment (FACIT-G), MIDOS, GHQ-12, Hospital Anxiety and Depression Scale (HADS), Rotterdam Symptom Checklist (RSCL), PEPS, Memorial Symptom Assessment Scale (MSAS-SF), and the RAI-PC were inadequately assessed because information on the measurement properties was incomplete or missing.

\section{Discussion}

Our systematic literature review identified 39 self-administered instruments measuring HRQoL mainly in patients with advanced cancer. None of the included studies reported sufficient information on psychometric properties of these measurement instruments according to the COSMIN criteria. Surprisingly, even basic psychometric properties such as construct validity and reliability were often inadequately tested. It appears that selecting an appropriate measurement instrument for testing construct validity and formulating specific hypotheses can be challenging. Furthermore, our findings show that adequate testing of responsiveness was not a priority in previous studies. PROMs are often used in clinical practice to monitor symptoms over time, it is therefore of great importance that a measurement instrument is responsive to changes. Despite incomplete information in the included studies, results of this review indicate that the EORTC QLQ-C15-PAL is an adequate instrument to measure HR in patients with advanced cancer. The EORTC QLQ-BM22, a module for patients with bone metastases, also appears to be suitable in this patient population. The EORTC QLQ-BM22 is a module and should be administered together with the EORTC QLQ-C30. Consequently, the measurement instrument is more extensive compared to the EORTC QLQ-C15-PAL. The length of a measurement instrument should be taken into account because there is little time for administration in clinical practice and a lower burden can foster compliance [99].

Due to medical advances, cancer is increasingly perceived as a chronic illness. Patients stretch the palliative phase by a longer survival and there is an increasing awareness to detect the palliative phase at an earlier stage when patients are relatively fit. The EORTC QLQ-C15-PAL may not be appropriate to administer in the beginning of the palliative phase due to its focus on symptoms at the end of life. When the EORTC QLQ-C15-PAL is administered in relatively healthy patients, a patients' actual HRQoL may be lower than what the EORTC QLQ-C15-PAL scores indicate and the EORTC QLQ-C30 will provide a more accurate reflection of a patients' HRQoL. The EORTC QLQ-C30 is the most commonly used disease-specific measure world-wide [100] and has been used in more than 3000 studies [101]. The routine use of the EORTC QLQ-C30 in clinical practice appears to improve physician-patient communication and HRQoL [102], but the implementation has its challenges (e.g. timing, frequency, interpretations of scores by health care professionals, and the absence of thresholds for clinical importance) [103]. Surprisingly, the present review showed that the psychometric quality of this measurement instrument has been examined many times but not adequately in patients with advanced cancer. Therefore, a thorough validation of the internal consistency, reliability, responsiveness, and interpretability of the EORTC QLQ-C30 in advanced cancer patients is advocated.

Another consideration regarding the reviewed HRQoL measurement instruments is that many of the instruments did not measure all aspects of HRQoL. Moreover, measurement instruments that only addressed one domain of HRQoL were excluded from our study. The spiritual domain is especially important at the end of life, but this domain was not often included in existing measurement instruments [28]. For instance, the EORTC QLQ-C15-PAL also did not include certain topics that appear to be relevant for patients in the end of life: Quality of care, Preparation for death, Spirituality or Transcendence [78, 90, 104-107]. The EORTC QLQC15-PAL was derived from the EORTC QLQ-C30 and the authors confirmed that existential or spiritual issues were mentioned by health care professionals and some patients as important additional topics to the measurement instrument. Therefore, the authors suggested that the EORTC QLQ-C15-PAL is supplemented by single items, modules, or questionnaires regarding spirituality when deemed necessary. This suggestion is especially valuable for clinical practice where the spirituality domain is not easily assessed in a regular doctor's appointment and many oncologists have not received specific training in palliative care.

\section{Practical implications}

For clinical practice it is important to monitor whether the latent construct that is being measured is represented by the selected instrument at the time of measurement and take the objective of measurement instrument into account 
when selecting an instrument. For instance, when interested in change over time one could argue that the EORTC QLQ-C15-PAL is less sensitive compared to the EORTC QLQ-C30 because it uses fewer items. However, sensitivity to change may also be improved by eliminating items that poorly represent the construct they were designed to measure [108]. In other words, improving measurement precision will enlarge sensitivity. Therefore, the EORTC QLQ-C15-PAL may actually be more sensitive to change over time when measuring HRQoL at the end of life in specific. However, because the EORTC QLQ-C15-PAL does not include items on spirituality the latent construct of HRQoL at the end of life is not fully measured. This reduces the sensitivity of the measurement instrument because the range where change can be detected over time is small [108]. Up to now, little is known about the measurement invariance of the QLQC15-PAL or EORTC QLQ-C30 in advanced cancer patients. Further validation to improve available information regarding minimal important differences and clinical relevance of differences in scores can aid interpretability in clinical practice [30]. PROMs have the potential to personalize care by identifying patients' needs but an accurate image of the patients' needs can only be achieved when administering the right measurement instrument at the right time for the right purposes.

This study has certain strength and limitations. It is important that the validation of instruments is performed in a consistent manner and evaluated as such. Using the COSMIN criteria in this review promoted a consistent evaluation. A limitation of this review is that there is no guarantee that our study selection procedure was sufficiently extensive. Even though references of included studies were checked, it is possible that certain validation studies were missed. Finally, this review only included measurement instruments that were not cancer site specific, meaning that the target population of the instrument was not focussed at patients with specific primary cancer sites. It is possible that for certain cancer sites, the EORTC QLQ-C15-PAL may not be the most adequate measure.

In conclusion, this review identified many self-administered instruments that measure $\mathrm{HRQoL}$ in patients with advanced cancer in clinical practice. Many of the existing measurement instruments have not yet been evaluated in an adequate manner, making it difficult to compare instruments. Considering the available information, the EORTC QLQ-C15-PAL and the EORTC QLQ-BM22 appeared to have best psychometric properties. However, there is no 'one size fits all', meaning that when selecting a measurement instrument in clinical practice it is important to take certain aspects into account such as the burden of administration and the objective of measurement (e.g. change over time). It is important that health care professionals possess upto-date knowledge on the quality of HRQoL measurement instruments to make an adequate selection in clinical practice. For instance, health care professionals should be aware that it is important to supplement existing measurement instruments with relevant items on spirituality or preparation of dying, depending on the patients' position within the palliative phase to accurately measure HRQoL. Validation of self-administered HRQoL measurement instruments is an important ongoing development because information on psychometric properties will enhance comparisons between instruments. This review contributes to improved clarity regarding the availability and quality of HRQoL measurement instruments for patients with advanced cancer and supports health care professionals in an adequate selection of suitable PROMs in advanced cancer patients in clinical practice. Being able to accurately and routinely measure HRQoL in patients with advanced cancer will stimulate the personalized health care approach leading to improved cancer care, clinical outcomes, and HRQoL.

Author contributions HF and NR participated in the design of the study. HF, JvR, and NR were involved in the data collection, analysis, and interpretation. JvR drafted the manuscript. All authors were involved in the critical revision of the manuscript and approved the final version of the manuscript.

Funding This work was supported by the Roparun Foundation.

\section{Compliance with ethical standards}

Conflict of interest The author(s) declared no potential conflicts of interest with respect to the research, authorship, and/or publication of this article.

Open Access This article is distributed under the terms of the Creative Commons Attribution 4.0 International License (http://creativeco mmons.org/licenses/by/4.0/), which permits unrestricted use, distribution, and reproduction in any medium, provided you give appropriate credit to the original author(s) and the source, provide a link to the Creative Commons license, and indicate if changes were made.

\section{References}

1. WHO.(2002). Definition palliative care, http://www.hoint/cance r/palliative/definition/en/. Accessed November 232016.

2. Temel, J. S., Greer, J. A., Muzikansky, A., et al. (2010). Early palliative care for patients with metastatic non-small-cell lung cancer. New England Journal of Medicine, 363(8), 733-742.

3. Coons, S. J., Rao, S., Keininger, D. L., \& Hays, R. D. (2000). A comparative review of generic quality-of-life instruments. Pharmacoeconomics, 17(1), 13-35.

4. Kaasa, S., \& Loge, J. H. (2003). Quality of life in palliative care: Principles and practice. Palliative Medicine, 17(1), 11-20.

5. Conroy, T., Bleiberg, H., \& Glimelius, B. (2003). Quality of life in patients with advanced colorectal cancer: What has been learnt? European Journal of Cancer, 39(3), 287-294.

6. Saeteren, B., Lindström, U., \& Nåden, D. (2011). Latching onto life: Living in the area of tension between the possibility 
of life and the necessity of death. Journal of Clinical Nursing, 20(5-6), 811-818.

7. Detmar, S. B., Aaronson, N. K., Wever, L. D., et al. (2000). How are you feeling? Who wants to know? Patients' and oncologists' preferences for discussing health-related quality-of-life issues. Journal of Clinical Oncology, 18(18), 3295-3301.

8. Mystakidou, K., Tsilika, E., Parpa, E., et al. (2008). Demographic and clinical predictors of spirituality in advanced cancer patients: A randomized control study. Journal of Clinical Nursing, 17(13), 1779-1785.

9. Edwards, A., Pang, N., Shiu, V., et al. (2010). The understanding of spirituality and the potential role of spiritual care in endof-life and palliative care: A meta-study of qualitative research. Palliative Medicine, 24(8), 753-770.

10. Winkelman, W. D., Lauderdale, K., Balboni, M. J., et al. (2011). The relationship of spiritual concerns to the quality of life of advanced cancer patients: Preliminary findings. Journal of Palliative Medicine, 14(9), 1022-1028.

11. Kimmel, P. L., Emont, S. L., Newmann, J. M., et al. (2003). ESRD patient quality of life: Symptoms, spiritual beliefs, psychosocial factors, and ethnicity. American Journal of Kidney Diseases, 42(4), 713-721.

12. Steinhauser, K. E., Christakis, N. A., Clipp, E. C., et al. (2000). Factors considered important at the end of life by patients, family, physicians, and other care providers. JAMA, 284(19), 2476-2482.

13. Etkind, S. N., Daveson, B. A., Kwok, W., et al. (2015). Capture, transfer, and feedback of patient-centered outcomes data in palliative care populations: Does it make a difference? A systematic review. Journal of Pain and Symptom Management, 49(3), 611-624.

14. Basch, E., Deal, A. M., Kris, M. G., et al. (2016). Symptom monitoring with patient-reported outcomes during routine cancer treatment: A randomized controlled trial. Journal of Clinical Oncology, 34(6), 557-565.

15. Fayers, P., \& Machin, D. (2008). Quality of life: The assessment, analysis and interpretation of patient-reported outcomes. Biometrics, 64(3), 996.

16. Kotronoulas, G., Kearney, N., Maguire, R., et al. (2014). What is the value of the routine use of patient-reported outcome measures toward improvement of patient outcomes, processes of care, and health service outcomes in cancer care? A systematic review of controlled trials. Journal of Clinical Oncology, 32(14), 1480-1501.

17. Takeuchi, E. E., Keding, A., Awad, N., et al. (2011). Impact of patient-reported outcomes in oncology: A longitudinal analysis of patient-physician communication. Journal of Clinical Oncology, 29(21), 2910-2917.

18. Marshall, S., Haywood, K., \& Fitzpatrick, R. (2006). Impact of patient-reported outcome measures on routine practice: A structured review. \ournal of Evaluation in Clinical Practice, 12(5), 559-568.

19. Howell, D., Molloy, S., Wilkinson, K., et al. (2015). Patientreported outcomes in routine cancer clinical practice: A scoping review of use, impact on health outcomes, and implementation factors. Annals of Oncology, 26(9), 1846-1858.

20. Bruley, D. K. (1999). Beyond reliability and validity: Analysis of selected quality-of-life instruments for use in palliative care. Journal of Palliative Medicine, 2(3), 299-309.

21. Hearn, J., \& Higginson, I. J. (1997). Outcome measures in palliative care for advanced cancer patients: A review. Journal of Public Health Medicine, 19(2), 193-199.

22. Jordhoy, M. S., Inger Ringdal, G., Helbostad, J. L., et al. (2007). Assessing physical functioning: A systematic review of quality of life measures developed for use in palliative care. Palliative Medicine, 21(8), 673-682.
23. Kaasa, S., \& Loge, J. H. (2002). Quality-of-life assessment in palliative care. Lancet Oncology, 3(3), 175-182.

24. Kirkova, J., Davis, M. P., Walsh, D., et al. (2006). Cancer symptom assessment instruments: A systematic review. Journal of Clinical Oncology, 24(9), 1459-1473.

25. Massaro, T., \& McMillan, S. C. (2000). Instruments for assessing quality of life in palliative care settings. International Journal of Palliative Nursing, 6(9), 429-433.

26. McMillan, S. C. (1996). Quality-of-Life Assessment in Palliative Care. Cancer Control, 3(3), 223-229.

27. Mularski, R. A., Dy, S. M., Shugarman, L. R., et al. (2007). A systematic review of measures of end-of-life care and its outcomes. Health Services Research, 42(5), 1848-1870.

28. Albers, G., Echteld, M. A., de Vet, H. C., et al. (2010). Evaluation of quality-of-life measures for use in palliative care: A systematic review. Palliative Medicine, 24(1), 17-37.

29. Mokkink, L. B., Terwee, C. B., Patrick, D. L., et al. (2010). The COSMIN checklist for assessing the methodological quality of studies on measurement properties of health status measurement instruments: An international Delphi study. Quality of Life Research, 19(4), 539-549.

30. Henoch, I., Axelsson, B., \& Bergman, B. (2010). The Assessment of Quality of life at the End of Life (AQEL) questionnaire: A brief but comprehensive instrument for use in patients with cancer in palliative care. Quality of Life Research, 19(5), 739-750.

31. Thavarajah, N., Ray, S., Bedard, G., et al. (2015). Psychometric validation of the Brain Symptom and Impact Questionnaire (BASIQ) version 1.0 to assess quality of life in patients with brain metastases. CNS Oncology, 4(1), 11-23.

32. Chang, V. T., Hwang, S. S., Kasimis, B., \& Thaler, H. T. (2004). Shorter symptom assessment instruments: The Condensed Memorial Symptom Assessment Scale (CMSAS). Cancer Investment, 22(4), 526-536.

33. Chow, E., Hird, A., Velikova, G., et al. (2009). The European Organisation for Research and Treatment of Cancer Quality of Life Questionnaire for patients with bone metastases: The EORTC QLQ-BM22. European Journal of Cancer, 45(7), $1146-1152$.

34. Chow, E., Nguyen, J., Zhang, L., et al. (2012). International field testing of the reliability and validity of the EORTC QLQ-BM22 module to assess health-related quality of life in patients with bone metastases. Cancer, 118(5), 1457-1465.

35. Zeng, L., Chow, E., Bedard, G., et al. (2012). Quality of life after palliative radiation therapy for patients with painful bone metastases: Results of an international study validating the EORTC QLQ-BM22. International Journal of Radiation Oncology Biology Physics, 84(3), 337-342.

36. Zeng, L., Chow, E., Zhang, L., et al. (2012). An international prospective study establishing minimal clinically important differences in the EORTC QLQ-BM22 and QLQ-C30 in cancer patients with bone metastases. Supportive Care in Cancer: Official Journal of the Multinational Association of Support Care Cancer, 20(12), 3307-3313.

37. Wong, E., Zhang, L., Kerba, M., et al. (2015). Minimal clinically important differences in the EORTC QLQ-BN20 in patients with brain metastases. Support Care Cancer, 23(9), 2731-2737.

38. Bedard, G., Zeng, L., Zhang, L., et al. (2016). Minimal important differences in the EORTC QLQ-C15-PAL to determine meaningful change in palliative advanced cancer patients. Asia Pacific Journal of Clinical Oncology, 12(1), 38-46.

39. Groenvold, M., Petersen, M. A., Aaronson, N. K., et al. (2006). The development of the EORTC QLQ-C15-PAL: A shortened questionnaire for cancer patients in palliative care. European Journal of Cancer, 42(1), 55-64. 
40. Bedard, G., Zeng, L., Zhang, L., et al. (2014). Minimal important differences in the EORTC QLQ-C30 in patients with advanced cancer. Asia Pacific Journal of Clinical Oncology, 10(2), 109-117.

41. Giesinger, J. M., Kieffer, J. M., Fayers, P. M., et al. (2016). Replication and validation of higher order models demonstrated that a summary score for the EORTC QLQ-C30 is robust. Journal of Clinical Epidemiology, 69, 79-88.

42. Aaronson, N. K., Ahmedzai, S., Bergman, B., et al. (1993). The European Organization for Research and Treatment of Cancer QLQ-C30: A quality-of-life instrument for use in international clinical trials in oncology. Journal of the National Cancer Institute, 85(5), 365-376.

43. Kaasa, S., Bjordal, K., Aaronson, N., et al. (1995). The EORTC core quality of life questionnaire (QLQ-C30): Validity and reliability when analysed with patients treated with palliative radiotherapy. European Journal of Cancer, 31A(13-14), 2260-2263.

44. McLachlan, S. A., Devins, G. M., \& Goodwin, P. J. (1998). Validation of the European Organization for Research and Treatment of Cancer Quality of Life Questionnaire (QLQ-C30) as a measure of psychosocial function in breast cancer patients. European Journal of Cancer, 34(4), 510-517.

45. Osoba, D., Zee, B., Pater, J., et al. (1994). Psychometric properties and responsiveness of the EORTC quality of Life Questionnaire (QLQ-C30) in patients with breast, ovarian and lung cancer. Quality of Life Research, 3(5), 353-364.

46. Petersen, M. A., Groenvold, M., Aaronson, N., et al. (2006). Item response theory was used to shorten EORTC QLQ-C30 scales for use in palliative care. Journal of Clinical Epidemiology, 59(1), 36-44.

47. Stromgren, A. S., Groenvold, M., Pedersen, L., et al. (2002). Symptomatology of cancer patients in palliative care: Content validation of self-assessment questionnaires against medical records. European Journal of Cancer, 38(6), 788-794.

48. Sharp, L. K., Knight, S. J., Nadler, R., et al. (1999). Quality of life in low-income patients with metastatic prostate cancer: Divergent and convergent validity of three instruments. Quality of Life Research, 8(5), 461-470.

49. Stiel, S., Psych, D., Kues, K., et al. (2011). Assessment of quality of life in patients receiving palliative care: Comparison of measurement tools and single item on subjective well-being. Journal of Palliative Medicine, 14(5), 599-606.

50. Hjermstad, M. J., Bergenmar, M., Fisher, S. E., et al. (2012). The EORTC QLQ-OH17: A supplementary module to the EORTC QLQ-C30 for assessment of oral health and quality of life in cancer patients. European Journal of Cancer, 48(14), 2203-2211.

51. Vivat, B., Young, T., Efficace, F., et al. (2013). Cross-cultural development of the EORTC QLQ-SWB36: A stand-alone measure of spiritual wellbeing for palliative care patients with cancer. Palliative Medicine, 27(5), 457-469.

52. Bedard, G., Zeng, L., Zhang, L., et al. (2013). Minimal clinically important differences in the Edmonton symptom assessment system in patients with advanced cancer. Journal of Pain and Symptom Management, 46(2), 192-200.

53. Chang, V. T., Hwang, S. S., \& Feuerman, M. (2000). Validation of the edmonton symptom assessment scale. Cancer, 88(9), 2164-2171.

54. Bush, S. H., Parsons, H. A., Palmer, J. L., et al. (2010). Singlevs. multiple-item instruments in the assessment of quality of life in patients with advanced cancer. Journal of Pain and Symptom Management, 39(3), 564-571.

55. Nekolaichuk, C. L., Maguire, T. O., Suarez-Almazor, M., et al. (1999). Assessing the reliability of patient, nurse, and family caregiver symptom ratings in hospitalized advanced cancer patients. Journal of Clinical Oncology, 17(11), 3621-3630.
56. Philip, J., Smith, W. B., Craft, P., \& Lickiss, N. (1998). Concurrent validity of the modified Edmonton symptom assessment system with the rotterdam symptom checklist and the brief pain inventory. Support Care Cancer, 6(6), 539-541.

57. Watanabe, S. M., Nekolaichuk, C. L., \& Beaumont, C. (2012). The Edmonton Symptom Assessment System, a proposed tool for distress screening in cancer patients: Development and refinement. Psychooncology, 21(9), 977-985.

58. Claessens, P., Menten, J., Schotsmans, P., \& Broeckaert, B. (2011). Development and validation of a modified version of the Edmonton Symptom Assessment Scale in a Flemish palliative care population. American Journal of Hospice and Palliative Medicine, 28(7), 475-482.

59. Krabbe, P. F., Peerenboom, L., Langenhoff, B. S., \& Ruers, T. J. (2004). Responsiveness of the generic EQ-5D summary measure compared to the disease-specific EORTC QLQ C-30. Quality of Life Research, 13(7), 1247-1253.

60. Ribaudo, J. M., Cella, D., Hahn, E. A., et al. (2000). Re-validation and shortening of the Functional Assessment of Anorexia/Cachexia Therapy (FAACT) questionnaire. Quality of Life Research, 9(10), 1137-1146.

61. Salsman, J. M., Beaumont, J. L., Wortman, K., et al. (2015). Brief versions of the FACIT-fatigue and FAACT subscales for patients with non-small cell lung cancer cachexia. Support Care Cancer, 23(5), 1355-1364.

62. Thavarajah, N., Bedard, G., Zhang, L., et al. (2014). Psychometric validation of the functional assessment of cancer therapybrain (FACT-Br) for assessing quality of life in patients with brain metastases. Support Care Cancer, 22(4), 1017-1028.

63. Lyons, K. D., Bakitas, M., Hegel, M. T., et al. (2009). Reliability and validity of the Functional Assessment of Chronic Illness Therapy-Palliative care (FACIT-Pal) scale. Journal of Pain and Symptom Management, 37(1), 23-32.

64. Zeng, L., Bedard, G., Cella, D., et al. (2013). Preliminary results of the generation of a shortened quality-of-life assessment for patients with advanced cancer: The FACIT-Pal-14. Journal of Palliative Medicine, 16(5), 509-515.

65. Gao, W., Stark, D., Bennett, M. I., et al. (2012). Using the 12-item General Health Questionnaire to screen psychological distress from survivorship to end-of-life care: Dimensionality and item quality. Psychooncology, 21(9), 954-961.

66. Le Fevre, P., Devereux, J., Smith, S., et al. (1999). Screening for psychiatric illness in the palliative care inpatient setting: A comparison between the Hospital Anxiety and Depression Scale and the General Health Questionnaire-12. Palliative Medicine, 13(5), 399-407.

67. McMillan, S. C., \& Mahon, M. (1994). Measuring quality of life in hospice patients using a newly developed Hospice Quality of Life Index. Quality of Life Research, 3(6), 437-447.

68. McMillan, S. C., \& Weitzner, M. (1998). Quality of life in cancer patients: Use of a revised Hospice Index. Cancer Practice, 6(5), 282-288.

69. Hopwood, P., Howell, A., \& Maguire, P. (1991). Screening for psychiatric morbidity in patients with advanced breast cancer: Validation of two self-report questionnaires. British Journal of Cancer, 64(2), 353-356.

70. Locke, D. E., Decker, P. A., Sloan, J. A., et al. (2007). Validation of single-item linear analog scale assessment of quality of life in neuro-oncology patients. Journal of Pain and Symptom Management, 34(6), 628-638.

71. Pratheepawanit, N., Salek, M. S., \& Finlay, I. G. (1999). The applicability of quality-of-life assessment in palliative care: Comparing two quality-of-life measures. Palliative Medicine, 13(4), 325-334.

72. Lua, P. L., Salek, S., Finlay, I., \& Lloyd-Richards, C. (2005). The feasibility, reliability and validity of the McGill Quality of Life 
Questionnaire-Cardiff Short Form (MQOL-CSF) in palliative care population. Quality of Life Research, 14(7), 1669-1681.

73. Cohen, S. R., \& Mount, B. M. (2000). Living with cancer: "Good" days and "bad" days-what produces them? Can the McGill quality of life questionnaire distinguish between them? Cancer, 89(8), 1854-1865.

74. Cohen, S. R., Mount, B. M., Tomas, J. J., \& Mount, L. F. (1996). Existential well-being is an important determinant of quality of life. Evidence from the McGill Quality of Life Questionnaire. Cancer, 77(3), 576-586.

75. Cohen, S. R., Mount, B. M., Strobel, M. G., \& Bui, F. (1995). The McGill Quality of Life Questionnaire: A measure of quality of life appropriate for people with advanced disease. A preliminary study of validity and acceptability. Palliative Medicine, 9(3), 207-219.

76. Cohen, S. R., Mount, B. M., Bruera, E., et al. (1997). Validity of the McGill Quality of Life Questionnaire in the palliative care setting: A multi-centre Canadian study demonstrating the importance of the existential domain. Palliative Medicine, 11(1), 3-20.

77. Webber, K., \& Davies, A. N. (2011). Validity of the memorial symptom assessment scale-short form psychological subscales in advanced cancer patients. Journal of Pain and Symptom Management, 42(5), 761-767.

78. Byock, I. R., \& Merriman, M. P. (1998). Measuring quality of life for patients with terminal illness: The Missoula-VITAS quality of life index. Palliative Medicine, 12(4), 231-244.

79. Schwartz, C. E., Merriman, M. P., Reed, G., \& Byock, I. (2005). Evaluation of the Missoula-VITAS Quality of Life Indexrevised: Research tool or clinical tool? Journal of Palliative Medicine, 8(1), 121-135.

80. Romero, M. M., Flood, L. S., Gasiewicz, N. K., et al. (2015). Validation of the National Institutes of Health Patient-Reported Outcomes Measurement Information System Survey as a Quality-of-Life Instrument for Patients with Malignant Brain Tumors and Their Caregivers. Nursing Clinics of North America, 50(4), 679-690.

81. Yost, K. J., Eton, D. T., Garcia, S. F., \& Cella, D. (2011). Minimally important differences were estimated for six PatientReported Outcomes Measurement Information System-Cancer scales in advanced-stage cancer patients. Journal of Clinical Epidemiology, 64(5), 507-516.

82. Antunes, B., Murtagh, F., Bausewein, C., et al. (2015). Screening for depression in advanced disease: Psychometric properties, sensitivity, and specificity of two items of the Palliative Care Outcome Scale (POS). Journal of Pain and Symptom Management, 49(2), 277-288.

83. Hearn, J., \& Higginson, I. J. (1999). Development and validation of a core outcome measure for palliative care: The palliative care outcome scale. Palliative Care Core Audit Project Advisory Group. Quality in Health Care, 8(4), 219-227.

84. Siegert, R. J., Gao, W., Walkey, F. H., \& Higginson, I. J. (2010). Psychological well-being and quality of care: A factor-analytic examination of the palliative care outcome scale. Journal of Pain and Symptom Management, 40(1), 67-74.

85. Muriel, A. C., Moore, C. W., Baer, L., et al. (2012). Measuring psychosocial distress and parenting concerns among adults with cancer: The Parenting Concerns Questionnaire. Cancer, 118(22), 5671-5678.

86. Chochinov, H. M., Hassard, T., McClement, S., et al. (2008). The patient dignity inventory: A novel way of measuring dignity-related distress in palliative care. Journal of Pain and Symptom Management, 36(6), 559-571.

87. Albers, G., Pasman, H. R., Rurup, M. L., et al. (2008). Analysis of the construct of dignity and content validity of the patient dignity inventory. Cancer, 112(11), 2509-2517.
88. Mack, J. W., Nilsson, M., Balboni, T., et al. (2008). Peace, equanimity, and acceptance in the cancer experience (PEACE): Validation of a scale to assess acceptance and struggle with terminal illness. Cancer, 112(11), 2509-2517.

89. Hadorn, D. C., Sorensen, J., \& Holte, J. (1995). Large-scale health outcomes evaluation: How should quality of life be measured? Part II-Questionnaire validation in a cohort of patients with advanced cancer. Journal of Clinical Epidemiology, 48(5), 619-629.

90. Steinhauser, K. E., Bosworth, H. B., Clipp, E. C., et al. (2002). Initial assessment of a new instrument to measure quality of life at the end of life. Journal of Palliative Medicine, 5(6), 829-841.

91. Steinhauser, K. E., Clipp, E. C., Bosworth, H. B., et al. (2004). Measuring quality of life at the end of life: Validation of the QUAL-E. Palliat Support Care, 2(1), 3-14.

92. Lo, C., Burman, D., Swami, N., et al. (2011). Validation of the QUAL-EC for assessing quality of life in patients with advanced cancer. European Journal of Cancer, 47(4), 554-560.

93. Shahidi, J., Bernier, N., \& Cohen, S. R. (2010). Quality of life in terminally ill cancer patients: Contributors and content validity of instruments. Journal of Palliative Medicine, 26(2), 88-93.

94. Steel, K., Ljunggren, G., Topinkova, E., et al. (2003). The RAIPC: An assessment instrument for palliative care in all settings. American Journal of Hospice and Palliative Medicine, 20(3), 211-219.

95. de Haes, J. C., van Knippenberg, F. C., \& Neijt, J. P. (1990). Measuring psychological and physical distress in cancer patients: Structure and application of the Rotterdam Symptom Checklist. British Journal of Cancer, 62(6), 1034-1038.

96. Janssen, S. J., Teunis, T., van Dijk, E., et al. (2017). Validation of the Spine Oncology Study Group-Outcomes Questionnaire to assess quality of life in patients with metastatic spine disease. Spine Journal, 17(6), 768-776.

97. de Jager Meezenbroek, E., Garssen, B., Van den Berg, M., et al. (2012). Measuring spirituality as a universal human experience: Development of the Spiritual Attitude and Involvement List (SAIL). Journal of Psychosocial Oncology, 30(2), 141-167.

98. Sharma, R. K., Astrow, A. B., Texeira, K., \& Sulmasy, D. P. (2012). The Spiritual Needs Assessment for Patients (SNAP): Development and validation of a comprehensive instrument to assess unmet spiritual needs. Journal of Pain and Symptom Management, 44(1), 44-51.

99. Steinmann, D., Schafer, C., van Oorschot, B., et al. (2009). Effects of radiotherapy for brain metastases on quality of life (QoL). Prospective pilot study of the DEGRO QoL working party. Strahlentherapie und Onkologie, 185(3), 190-197.

100. Garratt, A., Schmidt, L., Mackintosh, A., \& Fitzpatrick, R. (2002). Quality of life measurement: Bibliographic study of patient assessed health outcome measures. BMJ, 324(7351), 1417.

101. EORTC.(2017). Questionnaires. http://groups.eortc.be/qol/eortc -qlq-c30. Accessed March 122017.

102. Velikova, G., Booth, L., Smith, A. B., et al. (2004). Measuring quality of life in routine oncology practice improves communication and patient well-being: A randomized controlled trial. Journal of Clinical Oncology, 22(4), 714-724.

103. Wintner, L. M., Sztankay, M., Aaronson, N., et al. (2016). The use of EORTC measures in daily clinical practice-A synopsis of a newly developed manual. European Journal of Cancer, 68, 73-81.

104. Aspinal, F., Hughes, R., Dunckley, M., \& Addington-Hall, J. (2006). What is important to measure in the last months and weeks of life?: A modified nominal group study. International Journal of Nursing Studies, 43(4), 393-403. 
105. Teno, J. M., Byock, I., \& Field, M. J. (1999). Research agenda for developing measures to examine quality of care and quality of life of patients diagnosed with life-limiting illness. Journal of Pain and Symptom Management, 17(2), 75-82.

106. Stewart, A. L., Teno, J., Patrick, D. L., \& Lynn, J. (1999). The concept of quality of life of dying persons in the context of health care. Journal of Pain and Symptom Management, 17(2), 93-108.
107. Fok, C. C., \& Henry, D. (2015). Increasing the sensitivity of measures to change. Prevention Science, 16(7), 978-986.

108. Cocks, K., King, M. T., Velikova, G., et al. (2011). Evidencebased guidelines for determination of sample size and interpretation of the European Organisation for the Research and Treatment of Cancer Quality of Life Questionnaire Core 30. Journal of Clinical Oncology, 29(1), 89-96. 